\title{
Ovariohisterectomía en perras por NOTES híbrida transvaginal: comparación prospectiva de laparoscopía y cirugía abierta
}

\author{
Ovariohisterectomy in dogs by transvaginal hybrid NOTES: prospective comparison with \\ laparoscopic and open technique
}

\author{
MJ Luz ${ }^{a *}$, GS Ferreira ${ }^{a}$, CL Santos ${ }^{\mathrm{b}}$, RM Ramos ${ }^{\mathrm{a}}$, DF Vale ${ }^{\mathrm{a}}$, SN Ribeiro ${ }^{\mathrm{a}}$, MV Brun , R Zoron ${ }^{\mathrm{a}}$, ALA Oliveira ${ }^{\mathrm{a}}$ \\ ${ }^{a}$ Hospital Veterinário, Universidade Estadual do Norte Fluminense Darcy Ribeiro, RJ, Brasil. \\ ${ }^{b}$ Universidade Federal do Rio de Janeiro, RJ, Brasil. \\ 'Universidade Federal de Santa Maria, RS, Brasil.
}

\begin{abstract}
SUMMARY
The objective of this study was to compare the hybrid transvaginal NOTES (Natural Orifice Translumenal Endoscopic Surgery) with video-assisted surgery using two portals and open surgery for ovariohysterectomy $(\mathrm{OVH})$ in dogs. Twenty-four client-owned mixed-breed female dogs, weighing 5 to $19 \mathrm{~kg}$, presented for OVH at the hospital were assigned to three groups. Group 1 (H-NOTES): OVH using the technique of hybrid NOTES; Group 2 (LOVH): two portals laparoscopic OVH; Group $3(\mathrm{OOVH})$ : OVH using open technique. Surgical time, complications, and postoperative pain were compared according to surgical procedure. Operative time in NOTES group had an average of 58.25 minutes and was statistically similar to group LOVH (mean 70.15) and OOVH (mean 41.37 minutes). The NOTES group had significantly lower postoperative pain scores for groups LOVH and OOVH (Melbourne scale). Minor complications were found in all groups, and no animal died in consequence of the procedures. The hybrid NOTES technique for laparoscopic ovariohysterectomy in dogs is feasible, with advantages such as less postoperative pain compared to laparoscopic and open techniques and less surgical time for LOVH. Transvaginal hybrid NOTES OVH is safe and may be associated with less post-operative pain when compared to standard video-assisted surgery with two portals and open surgery.
\end{abstract}

Key words: NOTES, transvaginal access, ovariohisterectomy, laparoscopy.

\section{RESUMEN}

El objetivo de este estudio fue comparar la técnica de NOTES (Natural Orifice Translumenal Endoscopic Surgery) híbrida transvaginal, con la cirugía videoasistida usando dos portales y la cirugía abierta para ovariohisterectomía $(\mathrm{OVH})$ en perras. Para esto fueron utilizadas 24 perras sin raza definida, provenientes de la rutina del Hospital Veterinario. Las perras fueron separadas en tres grupos. Grupo 1 (H-NOTES): OVH usando la técnica de NOTES híbrida; Grupo2 (LOVH): OVH mediante técnica laparoscópica videoasistida; Grupo 3 (OOVH): OVH usando la técnica quirúrgica abierta convencional. El tiempo, las complicaciones quirúrgicas y el dolor postoperatorio fueron comparados entre los grupos. El tiempo de cirugía del grupo NOTES tuvo un promedio de 58,25 minutos y fue estadísticamente igual al grupo LOVH (promedio 70,15 minutos) y OOVH (promedio 41,37 minutos). El grupo NOTES tuvo significativamente el puntaje más bajo de dolor postoperatorio que los grupos LOVH y OOVH (según escala de Melbourne). Las complicaciones menores fueron encontradas en todos los grupos y ningún animal murió a consecuencia de los procedimientos. La técnica NOTES híbrida para ovariohisterectomía laparoscópica en perras es factible, con ventajas como menor dolor postoperatorio comparado con la técnica laparoscópica videoasistida y abierta, además menor tiempo quirúrgico que LOVH. La técnica NOTES híbrida transvaginal es segura y puede ser asociada con menos dolor postoperatorio comparada a la cirugía videoasistida con dos portales y la técnica convencional abierta.

Palabras clave: NOTES, acceso transvaginal, ovariohisterectomía, laparoscopía.

\section{INTRODUCCIÓN}

La cirugía laparoscópica es una nueva modalidad en cirugía veterinaria cada vez más común, debido a las ventajas que ofrece, como, menor trauma quirúrgico y potencialmente menos tazas de complicaciones quirúrgicas (Davison y col 2004).

Aceptado: 20.06.2013.

* Av. Alberto Lamego, 2000, CEP:28013-600, Campos dos Goytacazes, RJ, Brazil; mvmonicaluz@gmail.com
Un paso lógico en la evolución de la cirugía laparoscópica podría ser eliminar completamente las incisiones abdominales usando técnicas a través de orificios naturales como boca, ano o vagina. La Cirugía Endoscópica por Orificios Naturales (NOTES) puede evitar incisiones que crean un defecto en la pared abdominal y los riesgos postoperatorios, como herniación con riesgo de obstrucción intestinal, infección incisional y dolor (Mc Gee y col 2006, Sumiyama y Gostou 2006).

El concepto de NOTES transvaginal es nuevo y ha sido utilizado en un modelo porcino para realizar ne- 
frectomía y colecistectomía en el ámbito clínico humano (Clayman y col 2007, Pai y col 2007, Zorron y col 2007a Zorron y col 2007b, Kaouk 2009, Navarra y col 2009). Este acceso ha sido usado exitosamente en la realización de ovariohisterectomías $(\mathrm{OVH})$ en perras (Brun y col 2011). Freeman y col aplicaron la técnica de NOTES en perras para el procedimiento de ovariectomía transgástrica y sus resultados animaron el desarrollo de la ruta transvaginal para este procedimiento (Freeman y col 2009, Freeman y col 2010).

El procedimiento de NOTES híbrida (H-NOTES) implica el uso de la asistencia del laparoscopio para la visualización y retracción, pero con una disección realizada a través del orificio natural (Pai y col 2007, Zorron y col 2010). La NOTES híbrida transvaginal implica la colocación de un laparoscopio en la cicatriz umbilical para observar la segura introducción de un portal transvaginal, y subsecuentemente, el uso de instrumentos a través de ese portal para realizar la retracción, manipulación y finalmente la extracción de los tejidos (Zorron y Col 2007a).

El objetivo de este estudio es evaluar la técnica de NOTES transvaginal híbrida para OVH, comparando la técnica laparoscópica videoasistida usando dos portales y la cirugía abierta convencional. La hipótesis es que la OVH realizada por NOTES híbrida puede causar menos dolor y sufrimiento, proporcionando similares complicaciones postoperatorias y tiempo quirúrgico que la $\mathrm{OVH}$ por laparoscopía videoasistida.

\section{MATERIAL Y MÉTODOS}

El estudio fue aprobado por el Comité de Ética de la Universidad Estatal del Norte Fluminense (protocolo 26/2009). Todos los animales recibieron cuidados de acuerdo con los "Principios de Animales de Laboratorio" formulados por la National Society for Medical Research y la "Guide for the Care and Use of Laboratory Animals" preparada por la National Academy of Sciences, USA.

Veinticuatro perras presentadas para OVH de diferentes razas provenientes de la rutina del hospital veterinario fueron seleccionadas para la investigación. Ellas fueron divididas aleatoriamente en tres grupos: Grupo 1: H-NOTES (NOTES híbrida transvaginal), Grupo 2: LOVH (ovariohisterectomía laparoscópica videoasistida) y Grupo 3: cirugía abierta (OOVH). Los animales fueron ubicados en los grupos 1, 2 ó 3 por sorteo. Los propietarios fueron informados acerca de la innovación de la técnica, de las posibles complicaciones, conversiones a cirugía abierta, reoperaciones y beneficios y firmaron el consentimiento para la cirugía. Todos los procedimientos fueron realizados por el mismo cirujano.

Todas las perras fueron premedicadas con clorhidrato de fentanilo $(0,005 \mathrm{mg} / \mathrm{kg} \mathrm{IM})$ asociado con acepromacina $(0,05 \mathrm{mg} / \mathrm{kg} \mathrm{IM}) . \_$La anestesia fue inducida con clorhidrato de ketamina $(5 \mathrm{mg} / \mathrm{kg} \mathrm{IV})$ y diazepam $(0,5 \mathrm{mg} /$ kg IV). La anestesia epidural (clorhidrato de lidocaína con $2 \%$ de epinefrina $5 \mathrm{mg} / \mathrm{kg}$ ) fue realizada entre el espacio intervertebral de L7 y S1 inmediatamente después de la inducción anestésica. Luego de la intubación endotraqueal del animal, el mantenimiento anestésico fue obtenido con isoflurano.

\section{TÉCNICA QUIRÚRGICA}

Grupo H-NOTES. En este grupo la técnica de OVH fue aplicada por NOTES transvaginal. Las perras fueron fijadas en posición de decúbito dorsal, sometidas a previa limpieza de la vagina con povidona yodada al $0,1 \%$ y sondeadas. Fue realizada una incisión de $1 \mathrm{~cm}$ para insertar un trocar mediante la técnica de Hasson a 1-2 $\mathrm{cm}$ posterior a la cicatriz umbilical en línea alba. La cavidad abdominal fue insuflada con dióxido de carbono $\left(\mathrm{CO}_{2}\right)$ hasta alcanzar una presión oscilante de 12 a $14 \mathrm{mmHg}$. Un laparoscopio de $10 \mathrm{~mm}$ y $30^{\circ}$ fue introducido en la cánula y las estructuras abdominales fueron visualizadas. En el segundo portal (un trocar de 11 $\mathrm{mm}$ ) fue introducido a través de la vagina con auxilio de un obturador, bajo el cérvix, con monitorización por cámara para evitar daño visceral. Todo el equipo de disección fue colocado a través del segundo portal (figura 1). Con el paciente en decúbito lateral, el ovario fue suspendido y fijado a la pared abdominal a través de un anclaje externo hecho con aguja e hilo quirúrgicos, como fue descrito previamente (Brun y col 2009). El pedículo ovárico fue cauterizado con electrocauterio bipolar (figura 2) y los hilos que sostenían el ovario a la pared abdominal fueron retirados. Luego de la cauterización y

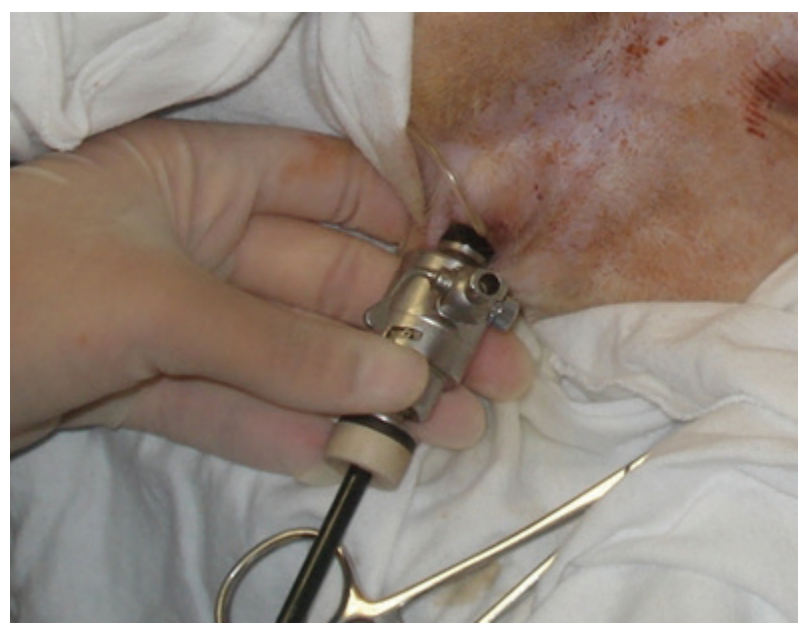

Figura 1. Abordaje transvaginal con laparoscopía control (grupo H-NOTES) en perras por ovariohisterectomía (OVH). Se observa la posición del trocarter de $6 \mathrm{~mm}$ para la introducción de la pinza laparoscópica.

Transvaginal approach with laparoscopic control (NOTES Group) in a bitch for ovariohysterectomy (OVH). Note the position of the $6 \mathrm{~mm}$ trocarter for inserting the laparoscopic instrument. 


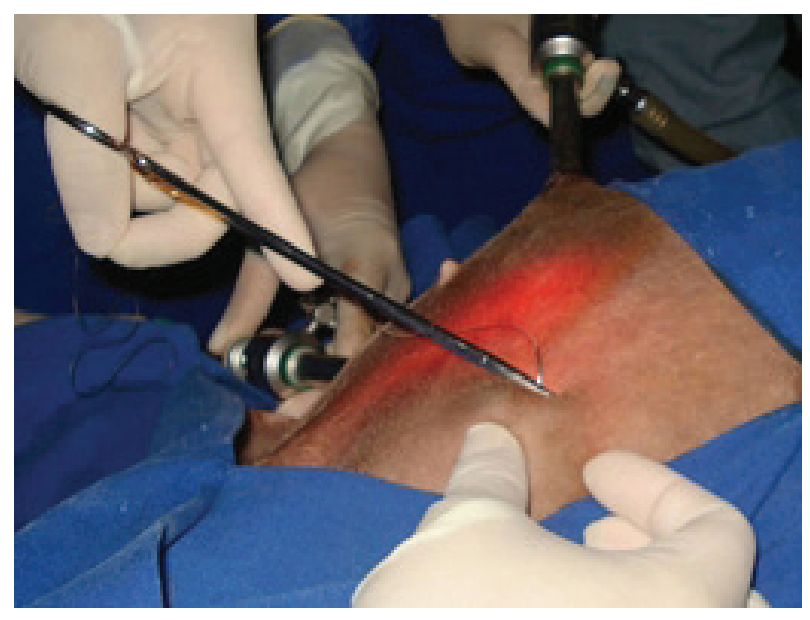

Figura 2. Fijación transparietal para la hemostasia del complejo arteriovenoso ovárico a través de electrocirugía.

Transparietal fixation for hemostasis of the ovary arteriovenous complex through electrosurgery.

la disección, el ovario derecho fue retirado caudalmente y exteriorizado a través de la incisión vaginal junto con el cuerno derecho, seguido por el ovario izquierdo y cuerno uterino. El cuerpo uterino fue exteriorizado mediante la incisión vaginal y la sutura realizada para cerrar la vagina fue hecha en la porción caudal del cérvix, antes de su disección, con hilo nylon 2-0 (figura 3). El útero fue colocado nuevamente en su posición anatómica luego de su ligadura y disección. La pared muscular y piel fueron suturados en dos planos con nylon 3-0. El resultado cosmético adecuado fue obtenido.

Grupo $L O V H$. La OVH asistida por laparoscopía fue realizada con dos portales. La técnica quirúrgica fue similar a la usada en el grupo H-NOTES, donde el primer portal fue introducido para la colocación del laparoscopio en la cicatriz umbilical, pero el segundo portal (con un trocar de $11 \mathrm{~mm}$ ) fue insertado sobre la línea media en el tercio caudal del abdomen, en la región prepúbica bajo directa visualización, para la colocación del trocar usado para pasar todas las pinzas de trabajo (Devitt y col 2005). Luego de la cauterización y resección del pedículo ovárico, el útero fue retirado en la misma secuencia del grupo H-NOTES. La sutura uterina fue realizada fuera de la cavidad abdominal, y el muñón uterino fue devuelto a su posición anatómica luego de su resección. La pared muscular y la piel fueron suturadas en dos planos con nylon 3-0.

Grupo OOVH. La ovariohisterectomía fue realizada usando laparotomía convencional. Las perras fueron colocadas en posición supina y exponiendo la línea media abdominal en el campo quirúrgico. Una incisión fue realizada en la línea alba inicialmente con bisturí y luego fue extendida con tijeras de Metzenbaum. El cuerno uterino izquierdo fue retirado de la cavidad con el dedo del cirujano y el pedículo ovárico fue ligado con doble ligadura

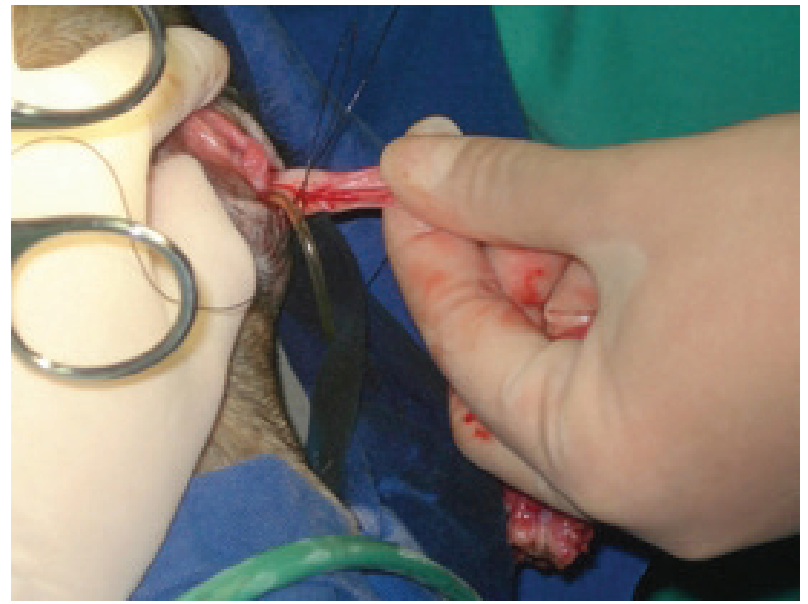

Figura 3. Exteriorización de la pieza quirúrgica después del procedimiento de H-NOTES transvaginal en una perra para OVH. Observar lasuturarealizadacaudalmente alaincisión delavagina. Exteriorization of surgical specimen after transvaginal $\mathrm{H}$ NOTES procedure in a bitch for OVH. Note the suture done caudally to the incision in the vagina.

nylon 2-0 y seccionado. Los mismos procedimientos fueron realizados en el pedículo ovárico derecho y cuerpo uterino. La pared abdominal fue suturada con nylon 3-0 en tres capas: muscular, tejido subcutáneo y piel.

Fue administrado ketoprofeno $(1,1 \mathrm{mg} / \mathrm{kg}$ vía oral, a cada 24 horas) durante tres días y cefalexina ( $30 \mathrm{mg} / \mathrm{kg}$ vía oral a cada 12 horas) durante siete días en todas las pacientes.

El tiempo quirúrgico fue registrado desde la primera incisión hasta el término de la sutura en piel.

\section{DOLOR POSTOPERATORIO}

Fue utilizada la escala de dolor de la Universidad de Melbourn (UMPS) (Tabla 1) (Mayhew y Cimino 2007). Los animales fueron evaluados por dos observadores ciegos, médicos veterinarios formados y con entrenamiento previo para evaluar el dolor de acuerdo con la escala de Melbourne. En esta escala los animales son evaluados antes de la realización de cualquier procedimiento para conocer el comportamiento normal del animal y eliminar errores durante la evaluación. Se les dió una puntuación de acuerdo con la presencia o ausencia de esos parámetros, donde 0 significa ausencia de dolor y 27 puntos el dolor máximo. Una puntuación menor a 4 es considerada como dolor mínimo (Firth y Haldane 1999). Entre 4 y 11, se considera dolor medio y por encima de 11 el dolor es grave, entonces debe ser administrada dosis extra de analgésico como clorhidrato de tramadol. Los animales fueron evaluados con la escala de dolor por uno de los dos observadores, en las horas $0,2,6$ y 24 postoperatorias, respectivamente llamadas T1, T2, T3 y T4. El tiempo 0 es inmediatamente antes de la recuperación de la anestesia epidural. 
Tabla 1. University of Melbourne Pain Scale (UMPS), used for pain evaluation.

Escala de dolor de la Universidad de Melbourne utilizada para evaluación del dolor.

\begin{tabular}{|c|c|c|}
\hline PARÁMETRO & DESCRIPCIÓN & PUNTUACIÓN \\
\hline \multirow[t]{2}{*}{ Pupila } & No dilatada & 0 \\
\hline & Dilatada & 2 \\
\hline \multirow{4}{*}{$\begin{array}{c}\text { Aumento o disminución de la frecuencia } \\
\text { cardiaca }\end{array}$} & $<20 \%$ & 0 \\
\hline & $>20 \%$ & 1 \\
\hline & $>50 \%$ & 2 \\
\hline & $>100 \%$ & 3 \\
\hline \multirow{4}{*}{$\begin{array}{c}\text { Aumento o diminución de la frecuencia } \\
\text { respiratoria }\end{array}$} & $<20 \%$ & 0 \\
\hline & $>20 \%$ & 1 \\
\hline & $>50 \%$ & 2 \\
\hline & $>100 \%$ & 3 \\
\hline \multirow[t]{2}{*}{ Temperatura rectal } & Dentro de los parámetros normales & 0 \\
\hline & Temperatura elevada & 1 \\
\hline \multirow[t]{2}{*}{ Salivación } & Sin salivación & 0 \\
\hline & Con salivación & 2 \\
\hline \multirow[t]{3}{*}{ Respuesta a la palpación del área afectada } & Sin reacción & 0 \\
\hline & Malestar durante la manipulación & 2 \\
\hline & Malestar durante y después de la manipulación & 3 \\
\hline \multirow[t]{4}{*}{ Actividad } & Durmiendo o comiendo & 0 \\
\hline & Despierto & 1 \\
\hline & Inquieto & 2 \\
\hline & Rodando & 3 \\
\hline \multirow[t]{4}{*}{ Estado mental } & Sumiso & 0 \\
\hline & Amigable & 1 \\
\hline & Desconfiado & 2 \\
\hline & Agresivo & 3 \\
\hline Postura & Acostado, cuidando el área afectada & 2 \\
\hline \multirow[t]{4}{*}{ Postura } & Decúbito lateral & 0 \\
\hline & Decúbito esternal & 1 \\
\hline & Sentado con la cabeza erguida & 1 \\
\hline & De pie con la cabeza agachada & 2 \\
\hline \multirow[t]{4}{*}{ Vocalización } & Sin vocalizar & 0 \\
\hline & Vocaliza cuando es manipulado & 2 \\
\hline & Vocalización intermitente & 2 \\
\hline & Vocalización contínua & 3 \\
\hline
\end{tabular}

\section{ANÁLISIS ESTADÍSTICO}

Para comparar la evolución temporal en la escala de dolor de Melbourne y el tiempo quirúrgico, fue utilizado el análisis ANOVA (One-way) de una vía para medidas independientes. La prueba de Tukey fue usada para comparar las diferencias entre grupos a cada tiempo, considerando una significancia estadística de $\mathrm{P}<0,05$. Todas las pruebas fueron realizadas utilizando el programa estadístico SISVAR (Microsoft Windows 4.0).

\section{RESULTADOS}

El abordaje transvaginal usado en el grupo H-NOTES fue fácil de realizar y permitió una buena visualización y manipulación de los órganos abdominales. No hubo conver- 
sión a técnica abierta o laparoscópica. La insuflación de la cavidad abdominal fue adecuada durante el procedimiento.

\section{TIEMPO QUIRÚRGICO}

El tiempo quirúrgico del grupo H-NOTES tuvo un promedio $58,25 \pm 15,9$ minutos y fue estadísticamente similar al grupo LOVH (promedio $70,15 \pm 19,7$ ) y OOVH (promedio 41,37 $\pm 11,3$ ). Sin embargo, el grupo OOVH tuvo tiempos operatorios estadísticamente menores comparados con el grupo LOVH, de acuerdo con el test de Tukey, $\mathrm{P}<0,01$ (figura 4).

\section{COMPLICACIONES}

Con respecto al grupo H-NOTES, dos animales presentaron sangrado vaginal leve. En uno de ellos, el sangrado ocurrió 48 horas después de realizado el procedimiento quirúrgico pero no requirió intervenciones adicionales. Otro animal tuvo disuria 24 horas luego de la cirugía y requirió administración de dipirona en dosis de $25 \mathrm{mg} / \mathrm{kg}$, también presentó intermitentes sangrados en pequeñas cantidades, que comenzaron 15 días después de la cirugía y fue aplicada la misma medicación del periodo postoperatorio inmediato, pero fue constatada leucocitosis discreta e infección urinaria en los exámenes realizados.

Fue observado enfisema subcutáneo postoperatorio en el área abdominal en un animal del grupo H-NOTES, pero desapareció sin necesidad de intervención en el postoperatorio inmediato.

Ocurrió daño visceral sólo en un animal del grupo HNOTES, ocasionando una lesión en la cápsula esplénica cuando se insertó el primer trocar, pero no fue necesaria cirugía correctiva porque durante la inspección no se encontró sangrado desde la lesión en la cavidad abdominal y el sangrado paró rápidamente.

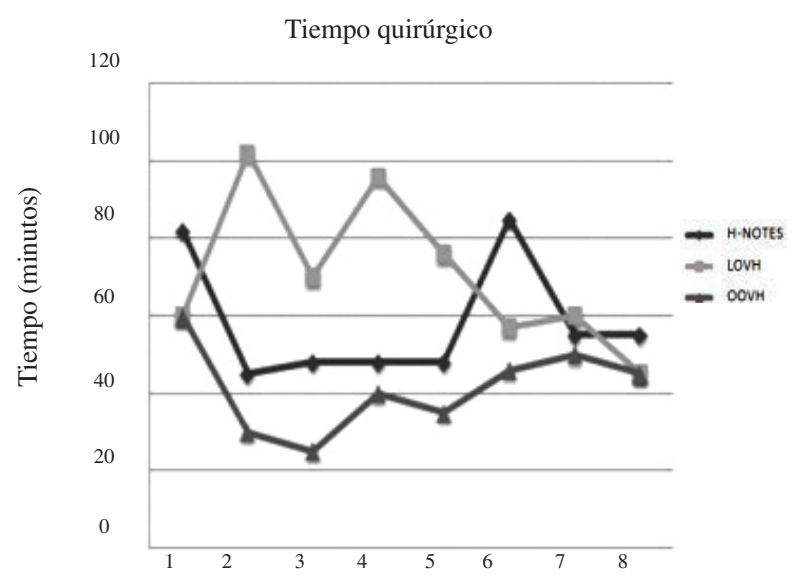

Figura 4. Disposición de los promedios de tiempos quirúrgicos de los tres grupos para el procedimiento de OVH.

Disposition of the average surgical time of the three groups for $\mathrm{OVH}$ procedure.
En el grupo LOVH, uno de los pacientes presentó sangrado en la pared abdominal muscular en el momento de insertar el segundo trocar. Este sangrado fue controlado con una ligadura del vaso muscular. Sin embargo, la perra tuvo sangrado severo y formación de hematomas 5 horas después de la cirugía, requiriendo nueva operación para control del sangrado.

En el grupo OOVH fue registrada una complicación en un animal. En este paciente hubo un sangrado menor en uno de los pedículos ováricos, que fue controlado mediante ligadura adicional en el mismo acto quirúrgico.

\section{DOLOR POSTOPERATORIO}

La media de los niveles de dolor (UMPS) de cada grupo en los cuatro períodos de tiempo estudiados son expuestos en la figura 5 . El rango de puntaje del grupo H-NOTES fue de 2,2 $\pm 1,2$ a 2,6 $\pm 0,9$; de $3,8 \pm 1,8$ a $6,5 \pm 4,1$ para el grupo LOVH y de $3,8 \pm$ 1,4 a $5,5 \pm 2,8$ en el grupo de cirugía abierta. El grupo H-NOTES tuvo significantemente menores puntajes comparados con el grupo LOVH y OOVH. Sin embargo, estos dos últimos grupos no difieren estadísticamente entre sí, según el test Tukey con $\mathrm{P}<0,05$.

\section{DISCUSIÓN}

El objetivo de este estudio fue comparar tres técnicas quirúrgicas para la realización de OVH. La técnica NOTES debe ser evaluada intensamente antes de su aplicación en medicina humana y los modelos animales siempre han sido una norma para estudiar los abordajes, beneficios, dolor postoperatorio y posibles complicaciones asociados a los procedimientos.

Promedios de las puntuaciones de color

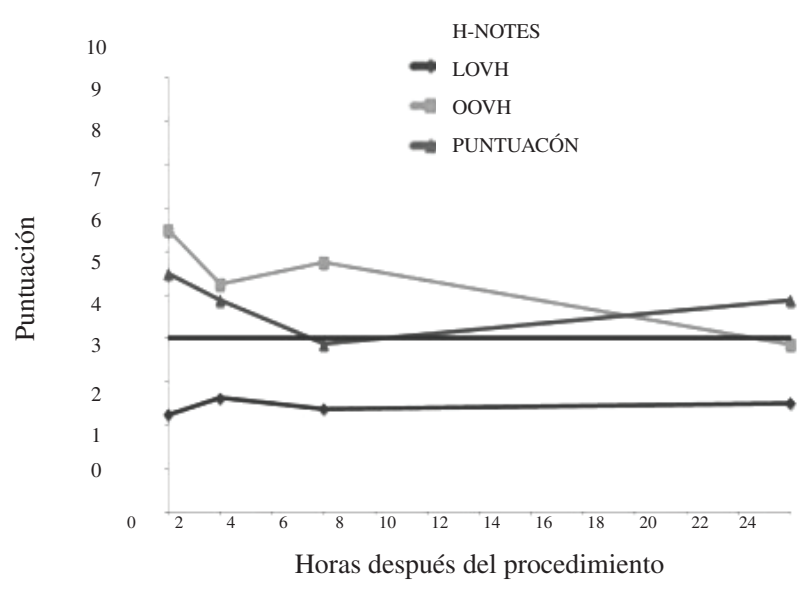

Figura 5. Disposición de los promedios de tiempos quirúrgicos de los tres grupos para el procedimiento de OVH.

Disposition of the average surgical time of the three groups for $\mathrm{OVH}$ procedure. 
Aún utilizando una técnica poco usual de H-NOTES transvaginal, el tiempo quirúrgico de este grupo fue estadísticamente similar al grupo LOVH. Este hecho puede darse debido a que la experiencia del cirujano es similar para ambas técnicas (cirugía laparoscópica y convencional para $\mathrm{OVH}$ ), sin causar una reducción en el tiempo del grupo H-NOTES influenciado por la experiencia. La técnica quirúrgica de NOTES híbrida usada para OVH, los instrumentos usados y la secuencia descrita probaron ser simples y fáciles de ser realizadas en perras. En 2009 fue encontrado un promedio de tiempo quirúrgico de 154 minutos en la técnica de NOTES transgástrica para ovariectomía (Freeman y col 2009). Según los autores, fue necesario primero realizar una gastropexia para la realización de la gastrotomía. Además, en algunas perras el endoscopio ubicado a la izquierda del estómago (quedó entre el omento) requiriendo una mayor insuflación e incrementando el tiempo quirúrgico de 30 a 45 minutos. Sin embargo, en un trabajo más reciente, estos autores lograron reducir el tiempo operatorio considerablemente luego de completar quirúrgicamente los pilotos el año anterior (Freeman y col 2010). Esto demuestra que, si la curva de aprendizaje se lleva a cabo, el procedimiento tiende a cumplirse más rápidamente y con mayor seguridad. El acceso de NOTES transvaginal híbrido probó ser técnicamente fácil de llevar a cabo y, además, el procedimiento se realizó en su totalidad sin mayores inconvenientes. El tiempo quirúrgico puede ser considerado pequeño comparado con la técnica de NOTES transgástrica usada para cirugía del sistema reproductor, debido a la fácil inserción de los trocares y el sencillo acceso a los ovarios y útero (Freeman y Col 2009, Freeman y Col 2010). Se cree que luego de haber superado la fase de curva de aprendizaje, el tiempo quirúrgico será menor.

La hemostasia del complejo arteriovenoso ovárico fue exitosa gracias a la cauterización con pinza de coagulación bipolar, sin la necesidad de utilizar clips o ligaduras. Cualquier sangrado mínimo desde el pedículo ovárico pudo ser controlado fácilmente usando electrocauterio bipolar. Las técnicas comúnmente usadas para ocluir los vasos ováricos son: electrocauterio bipolar, clips de titanio, ligadura con sutura y clamps vascular (Hansen 2003). Cuando son comparadas las técnicas para producir hemostasia para $\mathrm{OVH}$, el electrocauterio bipolar ofrece ventajas si es comparado con otros métodos de hemostasia en gatos (Schiochet y col 2009). El éxito con este tipo de hemostasia facilita el procedimiento operatorio, tornándolo más rápido y eficiente, pues el uso de clips y suturas, además de ser más costosos económicamente, también son más laboriosos de utilizar.

Solamente una perra perteneciente al grupo sometido a NOTES presentó daño en la cápsula esplénica, pero no fue necesaria intervención debido a la pequeña extensión de la lesión. Complicaciones como ésta, además de daño visceral y vascular, son citados en otro trabajo (Davidson y col 2004). Las lesiones esplénicas son descritas como la mayor complicación intraoperatoria, producida por el primer trocar o por la aguja de Veress. A pesar de este hecho, la técnica permitió una adecuada visualización de la lesión e inspección de la cavidad, sin requerir intervención, como fue reportado en un trabajo previo (Hansen 2003). Es importante tener en cuenta que las complicaciones citadas como el enfisema subcutáneo y el daño esplénico causado por la inserción del primer trocar encontrados en el grupo H-NOTES, ocurren comúnmente en la técnica laparoscópica, por eso, se debe tener bastante cuidado con la inserción de los trocares, para evitar este tipo de daños (McClaran 2009).

El uso de culdoscopio (también conocido como endoscopía transvaginal) usado por décadas para visualizar la cavidad abdominal y pélvica, es una técnica segura; pero tiene algunas complicaciones menores, por ejemplo han sido reportados casos de sangrado vaginal (Christian y col 2008). En este estudio, dos perras tuvieron sangrado vaginal en el tiempo postoperatorio. Una de ellas presentó reacción vaginal a la povidona yodada usada en la antisepsia de la vagina y de la pared abdominal, incrementando la sensibilidad a la manipulación durante la inserción del portal transvaginal. Sin embargo, esos cambios fueron reforzados por la sensibilidad particular del animal, ya que fue usada la misma concentración de povidona yodada en todas las perras, como fue mencionado anteriormente en otro trabajo (Hansen 2003). Quince días después de la cirugía, la paciente presentó secreción serosanguinolenta vaginal, la cual fue resuelta con la administración de antinflamatorios no esteroidales y antibióticos. Un resultado similar fue encontrado al respecto del sangrado postoperatorio vaginal cuando la ligadura de la vagina fue realizada craneal a la incisión. Pero cuando la incisión fue suturada completamente al mismo momento de la ligadura de la vagina, no se encontró sangrado en los pacientes.

En medicina humana, no se hallaron complicaciones postperatorias después de realizar colecistectomía en seis mujeres a través de NOTES transvaginal híbrida (Navarra y col 2009). En este estudio fue utilizado una óptica umbilical y un endoscopio flexible en la vagina. Otros autores usando la misma técnica, encontraron descarga vaginal en una de cuatro pacientes estudiadas después de realizar colecistectomía por NOTES transvaginal. Tal secreción fue atribuida a reacción al material de sutura usando en la vagina (Zorron y col 2008).

Por otro lado, es difícil llevar a cabo la evaluación del dolor en animales y han sido utilizados varios métodos para estimarlo. El uso de la UMPS en nuestro estudio fue satisfactorio, pues sólo requiere el empleo de un simple método numérico. El grupo H-NOTES mostró significativamente menores puntajes de dolor que el grupo LOVH. Este hecho está posiblemente relacionado al acceso y a la menor cantidad de incisiones abdominales, ya que en ambos grupos laparoscópicos H-NOTES y LOVH fueron usados los mismos instrumentos quirúrgicos, los mismos métodos de coagulación y las mismas presiones intraperi- 
toneales. El dolor postoperatorio en cirugía laparoscópica proviene del dolor visceral causado por la manipulación e irritación del diafragma debido a la presencia de $\mathrm{CO}_{2}$ (Pasqualucci 1996). Respecto a esto, se cree que son pocas las diferencias entre las técnicas, dado que la secuencia quirúrgica fue similar. Pero la inserción de los trocares en la pared abdominal fue también relacionados como causa del incremento del dolor y del sufrimiento postoperatorio. Por lo tanto, se asume que los bajos puntajes de dolor en el grupo H-NOTES fueron producto de la presencia de una única punción abdominal. Por otra parte, las incisiones en la pared abdominal causan pocos estímulos dolorosos, ya que los órganos abdominales son más sensibles a la expansión y a la compresión. Adicionalmente y en teoría, un menor número de punciones y el tamaño reducido de éstas, producirían un bajo estimulo doloroso, dos aspectos que impulsan el desarrollo de investigaciones en el área de cirugía a través de orificios naturales.

Son pocos los estudios relacionados al dolor en perras sometidas a OVH por NOTES. Freeman y col reportaron que los animales no tuvieron dolor en el procedimiento de NOTES luego de ovariectomía transgástrica (Freeman y Col 2009, Freeman y Col 2010). En ese trabajo, los autores evaluaron el dolor usando UMPS, pero también midieron cortisol sérico, glucosa, IL-6 (interleucina-6) proteína C-reactiva, y nivel de nocicepción a través de una Cuff de presión colocada alrededor del abdomen de los animales. Desafortunadamente, esos marcadores no fueron utilizados en este trabajo, pero el puntaje de dolor es considerado como una apropiada herramienta para tal evaluación (Freeman y col 2010, Mayhew y col 2007).

El uso de NOTES híbrida permite la visualización y la segura manipulación de los instrumentos para realizar la colpotomía. Serán necesarios más estudios sobre NOTES para establecer las rutas de acceso y las técnicas para mejorarla. Sin embargo, fue notoria la necesidad de desarrollar nuevas herramientas para el uso en NOTES y para facilitar el acceso por orificios naturales como boca, ano y vagina. Esto podría ocurrir por medio de más investigaciones, así que mayores inversiones en tecnología son esperadas.

La técnica de NOTES híbrida para OVH probó ser factible y segura en perras, con la ventaja de proporcionar menor dolor postoperatorio con similar tiempo quirúrgico en comparación con la técnica abierta y videoasistida. Las complicaciones de esta técnica pueden ser reducidas después de entrenamiento y avances en la curva de aprendizaje. El sangrado vaginal encontrado en dos perras puede ser evitado con la implementación de una ligadura caudal de la vagina antes de su recolocación anatómica.

\section{REFERENCIAS}

Brun M, MAM Silva, MW Ataíde JPS Feranti, FR Santos, LM Colomé, RL Guedes. 2009. NOTES híbrida na realização de ovariosalpingohisterectomia em 12 cadelas. Bras $J$ Video-Sur, Suppl 2, 70-71.
Brun M, MAM Silva, MB Mariano, AC Motta, LM Calomé, JPS Feranti, VL Pohl, MW Ataíde, RL Guedes, FR Santos. 2011. Ovariohysterectomy in a dog by a hybrid NOTES techinique. Can Vet J 52, 637-640.

Christian J, BF Barrier, D Schust, BW Miedema, K Thaler. 2008. Culdoscopy: A foundation for Natural Orifice Surgery Past, present, and future. J Am Coll Surg 207, 417-422.

Clayman RV, GN Box, JBA Abraham, HJ Lee, LA Deane, ER Sargent, NT Sguyen, K Chang, AK Tan, LE Ponsk, EM McDougall. 2007. Transvaginal Single-Port NOTES Nefhrectomy: Initial Laboratory Experience. J Endourol 21, 640-644.

Davidson EB, HD Moll, ME Payton. 2004. Comparison of laparoscopic ovariohysterectomy and ovariohysterectomy in dogs. Vet Surg 33, 62-69.

Devitt CM, RE Cox, JJ Hailey. 2005. Duration, complication, stress, and pain of open ovariohysterectomy versus a simple method of laparoscopic-assisted ovariohysterectomy in dogs. J Am Vet Med Assoc 227, 921-927.

Firth AM, SL Haldane. 1999. Development of a scale to evaluate postoperative pain in dogs. J Am Vet Med Assoc 214, 651-659.

Freeman LJ, EY Rahmani, S Sherman, MV Chiorean, DJ Selzer, PD Constable, P Snyder. 2009. Oophorectomy by natural orifice transluminal endoscopic surgery: feasibility study in dogs. Gastrointest Endosc 69, 1321-1332.

Freeman LJ, EY Rahmani, M Al-Haddad, S Sherman, MV Chiorean, DJ Selzer, PD Constable, PW Snyder. 2010. Comparison of pain and postoperative stress in dogs undergoing natural orifice transluminal endoscopic surgery, laparoscopic, and open oophorectomy. Gastrointest Endosc 72, 373-380.

Hansen B. 2003. Assessment of Pain in Dogs: Veterinary Clinical Studies. ILAR Journal 44, 197-205.

Kaouk JH, WM White, RK Goel, S Brethauer, S Crouzet, RR Rackley, C Moore, M Ingber, H Georges-Pascal. 2009. NOTES Transvaginal Nephrectomy: First Human Experience. Urology 74, 5-8.

Mayhew PD, D Cimino Brown. 2007. Comparison of three techniques for ovarian pedicle hemostasis during laparoscopic-assisted ovariohysterectomy. Vet Surg 36, 541-547.

McClaran JK, NJ Buote. 2009. Complications and Need for Conversion to Laparotomy in Small Animals. Vet Clin Small Anim 39, 941-951.

Mc Gee MF, MJ Rosen, J Marks, RP Onders, A Chak, A Faulx, VK Chen y J Ponsk. 2006. A Primer on Natural Orifice Transluminal endoscopic Surgery: Building a New Paradigm. Surg Innov 13, 86-93.

Navarra G, L Rando, G La Malfa, G Bartolotta, G Pracanica. 2009. Hybrid Transvaginal cholecystectomy: a novel approach. Am J Surg 197, 69-72.

Pai RD, DG Fong, ME Bundga, RD Odze, DW Rattner, CC Thompson. 2007. Transcolonic endoscopic cholecystectomy: a NOTES survival study in a porcine model (with video). Gastrointest Endosc 65, 428-434.

Pasqualucci A, V De Angelis, R Contardo, F Colo, A Terrosu, A Donini, A Pasetto, F Bresadola. 1996. Preemptive analgesia: intraperitoneal local anesthetic in laparoscopic cholecystectomy. A randomized double blind, placebo controlled study. Anesthesiology 85, 11-20.

Schiochet F, CAC Beck, APFF Silva, EA Contensini, MM 
Alievi, R Stedile, V Pinto, PH Yamazaki, DF Jurinitz, M Pellizari. 2009. Ovário-histerectomia laparoscópica em felinos hígidos: estudo comparativo de três métodos de hemostasia. Arq Bras Med Vet Zootec 61, 369-377.

Sumiyama K, CJ Gostout. 2006. Pilot study of the uterine horn as an in vivo appendicitis model for development of endoscopic transgastric appendectomy. Gastrointest Endosc 64, 808-812.

Zorron R, HL Fang, M Costa, M Soldan, A Lacerda, AJ Carneiro, CC Elia. 2007 ${ }^{\mathrm{a}}$. Alternative Techniques for Gastric Closure for NOTES. Gastrointest Endosc 65, 294.

Zorron R, M Filgueiras, L Maggioni, L Pombo, GL Carvalho, AL Oliveira. 2007b. NOTES Transvaginal Cholecystectomy: Report of the first case. Surg Innov 14, 278-283.
Zorron R, LC Maggioni, L Pombo, AL Oliveira, GL Carvalho, M Filgueiras. 2008. NOTES transvaginal cholecystectomy: preliminary clinical application. Surg Endosc 22, 542-547.

Zorron R, C Palanivelu, MP Galvão Neto, A Ramos, G Salinas, J Burghardt, L DeCarli, LH Sousa, A Forgione, R Pugliese, AJ Branco, TS Balashanmugan, C Boza, F Corcione, FD Avila, NA Gémez, PAG Ribeiro, S Martins, M Filgueiras, K Gellert, AW Branco, W Kondo, JI Sanseverino, JA de Sousa, L Saavedra, E Ramirez, J Campos, K Sivakumar, OS Rajan, PA Jategaonkar, M Ranagrajan, R Parthasarathi, P Senthilnathan, M Prasad, D Cuccurullo, V Muller. 2010. International Multicenter Trial on Clinical Natural Orifice Surgery - NOTES IMTN Study: Preliminary Results of 362 Patients. Surg Innov 17, 142-158. 